\title{
High prevalence of arthropathy in HTLV-I carriers on a Japanese island
}

\author{
Satoru Motokawa, Tomoko Hasunuma, Kazuo Tajima, Arthur M Krieg, Shin-ichiro Ito, \\ Katsuro Iwasaki, Kusuki Nishioka
}

Department of Orthopaedics, Nagasaki Chuo National Hospital, Nagasaki 856, Japan S Motokawa

Division of

Rheumatology, Institute of Medical

Science,

St Marianna

University,

Kawasaki 216, Japan

T Hasunuma

K Nishioka

Epidemiology

Division,

Aichi Cancer Institute,

Nagoya 464, Japan

K Tajima

Department of

Internal Medicine,

The University

of Iowa,

Iowa 52242-1081, USA

A $M$ Krieg

Izuhara Hospital, Nagasaki 857-23, Japan S Ito

Division of

Orthopedics,

Nagasaki University,

Nagasaki 852, Japan

K Iwasaki

Correspondence to:

Kusuki Nishioka, MD,

Division of Rheumatology,

Institute of Medical Science,

St Marianna University,

2-16-1 Sugao, Miyamae-ku,

Kawasaki-shi,

Kanagawa-ken 216, Japan

Accepted for publication

30 October 1995

\begin{abstract}
Objective-To determine the aetiological relationship between human $T$ cell leukaemia virus type-I (HTLV-I) and arthritis, by performing an epidemiological study of the inhabitants of Tsushima, an island northwest of the main island of Kyushu, Japan, which is an endemic area of HTLV-I.

Methods-A total of 7087 people underwent an annual health check, and those with arthropathy had further physical and radiological examinations by rheumatologists. The presence of HTLV-I antibody was determined by the particle agglutinin method, and integration of the proviral DNA in peripheral lymphocytes was confirmed by polymerase chain reaction.
\end{abstract}

Results-HTLV-I was positive in $26 \cdot 1 \%$ of inhabitants; the incidence increased to $37 \cdot 0 \%$ in patients with symptoms of polyarthritis. The prevalence of rheumatoid arthritis (RA) was significantly different between HTLV-I carriers $(0.56 \%)$ and non-carriers $(0 \cdot 31 \%)$. The stage of bone destruction in HTLV-I carriers with articular symptoms was milder than that in RA patients without HTLV-I. The relative risk of HTLV-I infection for polyarthritis was $1.66(p<0.05)$.

Conclusion-This is the first epidemiological report clarifying the association between HTLV-I and polyarthritis. Our results suggest that this viral infection has a relationship to RA.

(Ann Rheum Dis 1996; 55: 193-195)

Although the aetiology of rheumatoid arthritis (RA) has been studied in relation to several infectious agents such as Epstein-Barr virus and parvovirus, none of these viruses has been confirmed as the pathogenic agent in RA. We have studied human $T$ cell leukaemia virus type-I (HTLV-I), a member of the human retrovirus family, as a possible causative agent of RA. Several in vivo and in vitro studies investigating the pathogenesis of arthropathy have implicated the HTLV-I tax gene, a regulatory gene with a potent transcriptional activity on other genes, as a possible causative agent of RA. ${ }^{1-6}$

Our first report of HTLV-I associated arthropathy, ${ }^{1}$ revealed the need for an epidemiological field study to clarify the influence of HTLV-I infection on the incidence of RA in an area endemic for this virus. Tsushima is an island northwest of Kyushu, Japan, and is an area highly endemic for HTLV-I. It is an ideal area for epidemiological studies for several reasons, first, because the total population is approximately 46000 , which is sufficient for epidemiological studies, and second, because the island is sufficiently far from the mainland Kyushu that almost all the inhabitants visit the three main local clinics in Tsushima for primary medical care. Almost all local RA patients are treated in these three main hospitals, where we have conducted a study to evaluate the significance of serological and rheumatological findings among the inhabitants.

\section{Subjects and methods}

SUBJECTS

Of the 46064 inhabitants (1991 census) of Tsushima island, Nagasaki prefecture, Japan, 7087 inhabitants older than 30 years $(4588$ women, mean age 56.7 (SD 12.1) years; 2499 men, mean age 58.0 (12.0) years) voluntarily underwent an annual general health examination conducted by the local government. The population sample was screened for HTLV-I infection and the presence of arthritis. All individuals in the selected population sample who had arthropathy, including patients who were older than 30 years, had RA already diagnosed (according to the criteria of 1987 American College of Rheumatology $(\mathrm{ACR})^{7}$ ), and were receiving medication, were examined in the hospitals and assessed by physical and radiological examination by four rheumatologists. For several statistical comparisons, patients already diagnosed as having RA, but who did not participate in the health examination were included in the RA patient group.

The stage of bone destruction of the involved joints was evaluated by Steinbrocker's classification. ${ }^{8}$ These criteria classify bone destruction in patients with RA to the following four stages: stage $1=$ no erosion, with and without osteoporosis; stage $2=$ osteoporosis with and without subchondral bone destruction, no deformity; stage $3=$ osteoporosis with cartilage and bone destruction, presence of deformity such as ulnar deviation and subluxation; stage $4=$ fibrotic or bony ankylosis.

DETECTION OF HTLV-I INFECTION

Serum obtained was screened for HTLV-I antibody using the gelatin particle agglutination kit (Serodia-HTLV-I, Fuji-Rebio, 
Tokyo). Individuals found positive by this method were further confirmed as having HTLV-I infection by the polymerase chain reaction (PCR) method. DNA was extracted from peripheral blood lymphocytes and HTLV-I proviral DNA was amplified. Amplification was targetted to the $\mathrm{pX}$ region of HTLV-I and HLA-DQ $\beta$ as an internal control, as described previously. ${ }^{3}$

\section{STATISTICAL ANALYSIS}

Differences between the prevalence of HTLV-I in patients with and without RA were examined for significance using the Student's $t$ test. The prevalence of arthritis in HTLV-I carriers and non-carriers was determined by calculating the estimated number of HTLV-I carriers and non-carriers aged 30 years or more living in this island, using age specific positive rates for HTLV-I antibody: 7072 and 22 249, respectively. This difference was also examined for significance using the Student's $t$ test. The relative risk of arthritis in HTLV-I carriers was also calculated.

\section{Results}

PREVALENCE OF HTLV-I IN RA PATIENTS

Among the population examined, $26 \cdot 1 \%$ ( 1853 of 7087 ) were positive for HTLV-I infection. They comprised 537 of the 2499 men $(21.5 \%)$ and 1316 of the 4588 women $(28.7 \%)$ examined. Twenty one of the 7087 inhabitants were diagnosed as having symptomatic RA; nine of the 21 were found to have HTLV-I antibody, while the other 12 patients were negative for this antibody. In these 21 patients, five patients were newly diagnosed as RA (two were positive for HTLV-I and three were negative). For the analysis of the prevalence of HTLV-I infection in RA patients, patients with RA who were already diagnosed and treated at one of the three hospitals were also included. As a result, among the RA patients, $37 \%$ ( 41 of 111 , including seven of 22 men $(31.8 \%)$ and 34 of 89 women $(38.2 \%)$, $95 \%$ confidence interval (CI) 28.0 to $45 \cdot 9$ ) were HTLV-I carriers-a rate significantly greater than the overall carrier rate $(26 \cdot 1 \%)$ $(95 \%$ CI $25 \cdot 1$ to $27 \cdot 1)(\mathrm{p}<0 \cdot 05)$.

PREVALENCE OF ARTHRITIS IN HTLV-I CARRIERS AND NON-CARRIERS

As noted above, among 21 patients who were diagnosed as having RA in this epidemiological study, nine were positive for HTLV-I antibody while the remaining 12 were negative. The prevalence rates of RA among HTLV-I carriers and non-carriers were $0.49 \%$ (nine of 1853 ) and $0 \cdot 23 \%$ (12 of 5234 ), respectively. When we included those patients who were already diagnosed as RA and recalculated the prevalence using the estimated total population of the island, the prevalence of RA among HTLV-I carriers and non-carriers was estimated as approximately $0.56 \%$ (41 of 7072) and $0.31 \%$ (70 of 22249 ), respectively $(p<0.05)$. The odds ratio for arthritis in

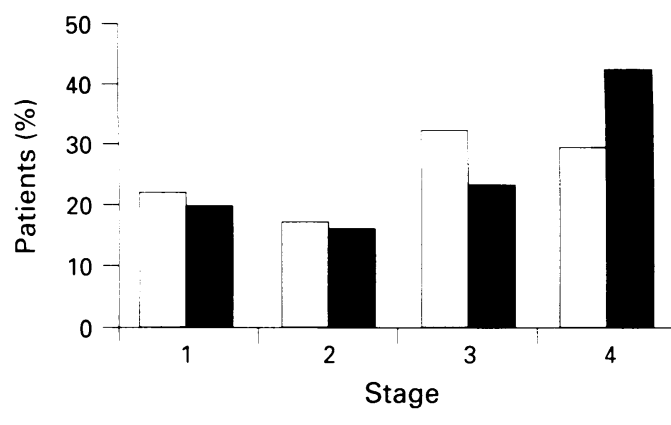

Comparative study of bone destruction stage in HTLV-I carriers ( $\square$ ) and non-carriers ( $\square$ ).

HTLV-I carriers, calculated from the age specific positive rate for HTLV-I, was 1.66 $\left(\chi^{2}=6.63, p<0.05\right)$. After adjustment for age and gender, the total odds ratio became 1.52 (men 1.58; women 1.39); however, this was not a statistically significant difference $(95 \%$ CI 0.82 to $1 \cdot 64$ ).

BONE DESTRUCTION IN HTLV-I CARRIER RA PATIENTS

As assessed by four rheumatologists using Steinbrocker's classification, there was a tendency for HTLV-I carrier patients to have a mild form of bone destruction that was statistically significant compared with noncarrier patients (figure), and joint swelling.

AGE OF HTLV-I CARRIER RA PATIENTS

Patients with RA who were carriers of HTLV-I were older than non-carriers (mean age of 41 HTLV-I positive RA patients $52 \cdot 1(13 \cdot 6)$ years; that of 70 HTLV-I negative RA patients 49.0 (12.9) years); however, this was not a statistically significant difference.

COMPARISON OF POSITIVITY OF EACH ITEM OF THE ACR CRITERIA

There was no significant difference in positivity of each of the ACR criteria in RA patients with and without HTLV-I (table).

\section{Discussion}

Since our first report of HTLV-I associated arthropathy, ${ }^{1}$ we have demonstrated the pathological role of HTLV-I in synovial proliferation in vitro and in vivo, using human synovial cells obtained from HTLV-I arthropathy patients, and experimentally in the transgenic mouse, ${ }^{2-6}$ and have also shown that the tax gene of this virus is responsible for synovial cell proliferation in vitro. However, it has been difficult to

\section{Positivity of ACR criteria in patients with rheumatoid arthritis positive (+) or negative (-) for HTLV-I virus}

\begin{tabular}{lcc}
\hline Criterion & $\begin{array}{c}H T L V-I(+) \\
(\%)\end{array}$ & $\begin{array}{c}H T L V-I(-) \\
(\%)\end{array}$ \\
\hline Morning stiffness & $87 \cdot 8$ & $90 \cdot 0$ \\
Arthritis of three or more joints & 100 & 100 \\
Arthritis of hand joints & 100 & 100 \\
Symmetric arthritis & 100 & $98 \cdot 6$ \\
Rheumatoid nodules & $87 \cdot 8$ & $91 \cdot 4$ \\
Serum rheumatoid factor & $58 \cdot 5$ & $68 \cdot 6$ \\
Radiographic changes & $80 \cdot 4$ & $81 \cdot 4$ \\
\hline
\end{tabular}


determine the epidemiological role of HTLV-I in RA because of the clinical diversity of this disease, and a large population study in an area endemic for HTLV-I was necessary. Tsushima island is an area highly endemic for HTLV-I, and provided an appropriate number of patients for study, from a population situated far enough away from the mainland of Japan to make it distinct.

In this study, the prevalence of HTLV-I carriers in RA patients was significantly greater than the prevalence estimated for this island. Moreover, the prevalence of RA in HTLV-I carriers in the island was greater than the estimated prevalence of RA in Japan $(0.49 \%)$ as reported by the Ministry of Health and Welfare, Japan (Matsuda et al, in preparation). In this study, the odds ratio for arthritis between HTLV-I carriers and non-carriers was 1.66; after adjustment for age and gender, this became $1.52(95 \%$ CI 0.82 to $1 \cdot 76)$. The reason for the failure of the results to attain statistical significance may be that the age of the HTLV-I carrier population was rather greater than that of the non-carrier population. Overall, however, these data suggest an association of HTLV-I with RA.

Comparison of the positivity of each ACR criterion between HTLV-I carriers and noncarriers revealed a lack of significant difference. However, comparison of the severity of bone and joint destruction in patients with and without HTLV-I revealed that HTLV-I infection tended to be associated with a milder bone destruction. Furthermore, the age at onset of articular symptoms was greater in HTLV-I carrier patients than in non-carrier patients. These data are in agreement with those from our first description of HTLV-I associated arthropathy. ${ }^{16}$

Is the incidence of RA greater in Japan or the Caribbean area because of the high prevalence of HTLV-I? Even though Japan is one of the areas endemic for HTLV-I, the HTLV-I carrier rate in 1987 was less than $1 \%$, and probably less than that in the present study. We believe that, because the positive rate is very small, it will not affect the prevalence of RA-a conclusion based on the fact that the epidemiological study was conducted in a socially semiclosed and highly endemic area of HTLV-I.
Several immunological disorders such as myelopathy, Sjögren's syndrome, uveitis, and bronchopneumonitis are considered to be related to HTLV-I..$^{9-14}$ Recently, Terada et al revealed a high prevalence of HTLV-I among patients with Sjögren's syndrome. ${ }^{15}$ These findings suggest that HTLV-I is one of the pathogenic retroviruses in immune disorders, ${ }^{15}$ and together with our present findings, confirm that HTLV-I is related to several immune disorders.

In conclusion, this is the first report demonstrating that HTLV-I infection is a risk factor for the development of RA.

This study was supported by a grant from the Japanese Ministry of Health and Welfare.

1 Nishioka K, Maruyama I, Maruyama Y, Kitajima I, Nakajima $Y$, Osame $M$. Chronic inflammatory arthropathy associated with HTLV-I. Lancet 1989; 1: 441

2 Iwakura $\mathrm{Y}$, Tosu $\mathrm{M}$, Yoshida $\mathrm{E}$, et al. Induction of inflammatory arthropathy resembling rheumatoid arthritis in mice transgenic for HTLV-I. Science 1991; 253: 1026-8.

3 Kitajima I, Yamamoto K, Sato K, et al. Detection of human $T$ cell lymphotropic virus type I proviral DNA and its gene expression in synovial cell in chronic inflammatory arthropathy. F Clin Invest 1991; 88: 1315-22.

4 Nakajima $T$, Aono $H$, Hasunuma $T$, et al. Overgrowth of human synovial cells driven by the HTLV-I tax gene. h Clin Invest 1991; 88: 1315-22.

5 Hasunuma T, Nakajima T, Aono $\mathrm{H}$, et al. Establishment and characterization of synovial cell clones with integrated human T-cell leukemia virus type-I. Clin Immunol Immunopathol 1994; 72: 90-7.

6 Sato K, Maruyama I, Maruyama Y, et al. Arthritis in patients infected with human T lymphotropic virus type-I. Arthritis Rheum 1991; 34: 714-21.

7 Arnett F C, Edworthy S M, Bloch D A, et al. The American Rheumatism Association 1987 revised criteria for the classification of rheumatoid arthritis. Arthritis Rheum 1988; 31: 315-24.

8 Steinbrocker O, Traeger C H, Batterman R C. Therapeutic criteria in rheumatoid arthritis. $\mathcal{F} A M A$ 1949; 140: criteria

9 Osame M, Usuku K, Izumo S, et al. HTLV-I associated myelopathy, a new clinical entity. Lancet 1986; i: 1031-2.

10 Gessain A, Barin F, Vernant J C, Gout O, Maurs L, Calender A. Antibodies to human T-lymphotropic virus type I in patients with tropical spastic paraparesis. Lancet 1985; ii: 407-10.

11 Eguchi K, Matsuoka N, Ida H, et al. Primary Sjögren's syndrome with antibodies to HTLV-I: clinical and

12 Green J E, Hinrichs S H, Vogel J, Jay G. Exocrinopathy resembling Sjögren's syndrome in HTLV-I tax transgenic resembling Sjögren's syndrom
mice. Nature 1989; 341: 72-4.

13 Mochizuki M, Watanabe T, Yamaguchi K. HTLV-I uveitis: a distinct clinical entity caused by HTLV-I. fpn $\mathcal{\jmath}$ Cancer Res 1992; 83: 236-9.

14 Vernant J C, Buisson G, Magdeleine J, et al. T-lymphocyte alveolitis, tropical spastic paraparesis, and Sjögren's syndrome. Lancet 1988; i: 177.

15 Terada K, Katamine S, Eguchi K, et al. Prevalence of serum and salivary antibodies to HTLV-I in Sjögren's syndrome. Lancet 1994; 344: 1116-9. 